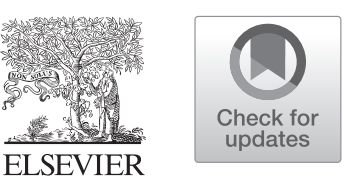

\title{
Impact of Pancreatic Autoantibodies in Pancreas Graft Survival After Pancreas-Kidney Transplantation
}

\author{
Nicole Pestana ${ }^{a, *}$, Jorge Malheiro ${ }^{b}$, Filipa Silva $^{b}$, Andreia Silva $^{c}$, Catarina Ribeiro $^{\mathrm{d}}$, Sofia Pedroso ${ }^{\mathrm{b}}$, \\ Manuela Almeida $^{\mathrm{b}}$, Leonídio Dias ${ }^{\mathrm{b}}$, António Castro Henriques ${ }^{\mathrm{b}}$, and La Salete Martins ${ }^{\mathrm{b}}$

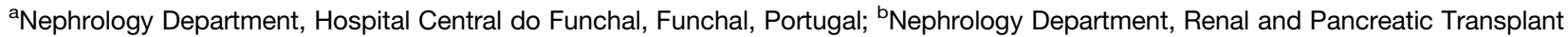

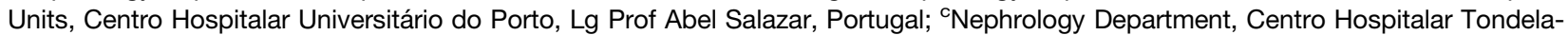 \\ Viseu, Viseu, Portugal; and dNephrology Department, Centro Hospitalar Vila Nova de Gaia/Espinho, Vila Nova de Gaia, Porto, Portugal
}

\begin{abstract}
Background. In simultaneous pancreas-kidney transplantation (SPKT), persistence or recurrence of pancreatic autoantibodies (PAs) has been associated with pancreas graft (PG) autoimmune-driven injury. Our aim was to analyze the impact of PAs on PG survival.

Methods. Between January 1, 2000, and December 31, 2017, we studied 139 patients with post-SPKT anti-glutamic acid decarboxylase (GAD) autoantibody. Alloimmune (ALI) events were defined as PG rejection and/or de novo donor-specific antibodies (DSA). Hence, 3 groups were defined: patients without ALI events or anti-GAD $(n=42)$, those with ALI events $(n=14)$, or those only with autoimmune events (positive for anti-GAD and no ALI events; $\mathrm{n}=83$ ).

Results. Male sex was predominant $(\mathrm{n}=72,52 \%)$. Median age was 35 years (interquartile range: 31-39) and median follow-up was 6-7 years (interquartile range: 4.1-9.2). Regarding anti-GAD positivity post-SPKT $(\mathrm{n}=90,65 \%)$, no differences were observed concerning age, sex, anti-HLA antibodies, HLA mismatch number and de novo DSA. ALI events were present in $10 \%(\mathrm{n}=14)$. PG survival 15 years post-SPKT was better in patients without immune events (96\%) followed by those with ALI (69\%) and autoimmune events (63\%) $(P=.025)$. Anti-GAD was associated to higher annualized mean $\operatorname{Hb} 1 \mathrm{AC}(P=.006)$ and lower mean C-peptide $(P=.013)$. According to pre- and post-SPKT anti-GAD status, conversion from negative to positive was associated to worse $(63 \%)$ 10-year PG survival $(P=.044)$, compared to persistence of negative $(100 \%)$ or positive anti-GAD $(88 \%)$. Anti-islet cell and anti-insulin autoantibodies had no impact.

Conclusion. Anti-GAD presence post-SPKT was associated to higher pancreas disfunction and lower PG survival. De novo anti-GAD seems to offer a particular risk of PG failure.
\end{abstract}

G ROWING evidence in the last 2 decades has been consolidating the concept that insulin-dependent diabetes mellitus (IDDM) is a chronic, destructive organ-specific autoimmune (AI) disorder. Sutherland et al [1] documented IDDM recurrence in pancreas transplant back in 1984. The first described cases were from HLA identical brothers with minimal immunosuppression [2]. These observations led to the hypothesis that recurrence of disease (AI isletitis leading to diabetes) was a major histocompatibility complex-restricted phenomenon.

$0041-1345 / 20$

https://doi.org/10.1016/j.transproceed.2020.02.035
Consecutive studies demonstrated that AI recurrence can occur independently of HLA match [3] and regardless of adequate immunosuppression [4]. Nevertheless, improved immunosuppression decreased the incidence of immunologic failures of whole pancreas grafts (PGs), a process

*Address correspondence to Maria Nicole Nunes Pestana, Nephrology Department, Hospital Central do Funchal, Avenida Luís de Camões 6180, 9000-177, Funchal, Portugal. Tel: +351291705600. E-mail: nicole.pest@gmail.com

(c) 2020 Elsevier Inc. All rights reserved. 230 Park Avenue, New York, NY 10169 
usually categorized as chronic rejection. Growing evidence indicates that chronic islet autoimmunity may eventually lead to recurrent diabetes and PG failure. It is reported that recurrent IDDM explains $50 \%$ of the immunologic failures, while the other $50 \%$ is attributed to chronic graft rejection [5].

IDDM recurrence is caused by the presence of inflammatory T-cell infiltrate targeting beta cells (insulitis) and sparing exocrine tissue, with no evidence of acute or chronic graft rejection. The presence of pancreatic autoantibodies (PAs), anti-islet cell (ICA), anti-glutamic acid decarboxylase (GAD), anti-insulin autoantibodies (IAA), anti-tyrosine phosphatase (IA2) and anti-tyrosine phosphatase (anti-ZnT8) is currently used as a noninvasive screening tool for follow-up (FU) of pancreas transplant, assuming that recurrence or rise of these autoantibodies titers are potential indicators of IDDM recurrence. It is unknown if these antibodies have a pathogenic action per se or are simply the result of a pancreatic lesion [6]. Nevertheless, there is undoubtedly an association between the presence of PAs and pancreas beta cells dysfunction after SKPT [7,8], being the majority of these antibodies detected in patients with biopsy specimens confirming AI recurrence. Positive PAs after simultaneous pancreas-kidney transplantation (SPKT) may never occur or it may be intermittent or persistent. Unfortunately, the real influence of PAs in PG dysfunction and survival is still controversial. Some authors have observed the association between recurrence of PA and poor pancreas survival $[9,10]$, while others did not find a relationship [11,12].

This study provides a thorough analysis of the PAs and their impact on recurrent autoimmunity and PG survival.

\section{METHODS}

All 211 consecutive adult patients who received a SPKT between January 2000 and December 2017 with more than 6 months of FU were investigated. All SPKT were performed at Renal and Pancreatic Units of Centro Hospitalar Universitário Porto, Portugal. Patients received induction therapy using a polyclonal antithymocyte globulin, $3 \mathrm{mg} / \mathrm{kg}$ for 5 to 7 days, and had similar triple maintenance immunosuppression, consisting of oral tacrolimus, mycophenolate mofetil and prednisolone. Moreover, cytomegalovirus prophylaxis was always performed for at least 6 months with oral valganciclovir. In terms of surgical technique, pancreas transplants were performed using a systemic-enteric drainage.

At initial evaluation, 72 patients were excluded as they had no consistent anti-GAD assessment post-SPKT. Hence, the final study cohort comprised the remaining 139 SPKT patients. All patients were followed from time of transplant until death or graft failure until December 31, 2018. Death-censored PG survival was determined from the time of SPKT until pancreas failure, patient death, or end of FU.

Blood samples were systematically collected to prospectively measure PAs. Screening was performed during the pretransplant evaluation; on admission day or in the early days thereafter; after transplantation at 6 months, 12 months; and then once per year after SPKT. During FU, a median of 4 screening samples (range, 26) per patient were performed. Anti-GAD were measured using the radio-ligand assay CentAK anti-GAD65 (MEDIPAN GMBH, Berlin, Germany). ICA were determined by enzyme-linked immunosorbent assay test, using the Isletest-ICA (BIOMERICA, Irvine, CA, United States). IAA were measured using the radioimmunoassay RiaRSRIAA (RSR Limited, Cardiff, United Kingdom). Patients were considered positive for PA when anti-GAD antibodies were $>1.45 \mathrm{U} / \mathrm{mL}$, ICA $>1.05$, and IAA $>0.4 \mathrm{U} / \mathrm{mL}$. The normal range for glycated hemoglobin (HbA1c) was $3.8 \%$ to $5.6 \%$ (18-38 $\mathrm{mmol} / \mathrm{mol}$ ) and for C-peptide $1.1 \mathrm{ng} / \mathrm{mL}$ to $4.4 \mathrm{ng} / \mathrm{mL}$. Continuous data were described using mean and standard deviation (SD) or median and interquartile range (IQR), and categorical data were expressed as number (and percentages). Categorical data including demographic, clinical, and immunologic features were compared using Pearson $\chi^{2}$ test or the Fisher exact test, as appropriate. Continuous variables were compared with Student $t$ test or MannWhitney $U$ test, as appropriate. Annualized mean $\mathrm{HbA} 1 \mathrm{c}$ and $\mathrm{C}$ peptide values were assessed by univariate linear mixed regression model that imputed subject-specific random effects (no intercept and slope time in years) on an unstructured covariance matrix. The dependent variable was all $\mathrm{HbA1c}$ and C-peptide measurements, and the independent variable (anti-GAD post-SPKT status) was entered as 2-way interaction term between them and the time (in years) variable. Graft survival curves were done using Kaplan-Meier method and compared by log-rank test. A two-sided $P$ value $<.05$ was considered as statistically significant. Statistical calculations were performed using STATA/MP, version 15.1 (Stata Corp, College Station, TX, United States).

\section{RESULTS}

\section{Study Population}

Studied population median FU was 6 to 7 years (4.1-9.2). The incidence of rejection and/or de novo donor-specific antibody (DSA) was $10 \%(\mathrm{n}=14)$. Seven patients died. PG loss occurred in 16 patients.

\section{Autoimmunity: Prospective Analysis of Pancreatic Antibodies}

Of the 139 SPKT patients, $19 \%$ were positive for anti-GAD antibodies before transplantation; $11 \%$ were ICA-positive; and $24 \%$ were IAA-positive. IAA $(P=.470)$ and ICA $(P=.945)$ did not reach significant difference concerning PG survival. Anti-GAD was the only antibody that showed impact on PG outcomes $(P=.001)$. Consequently, the patients were separated into 2 groups based on the presence $(n=90)$ or absence $(n=49)$ of anti-GAD after SPKT. Comparison of baseline characteristics of global cohort and according to anti-GAD status post-SPKT is presented in Table 1.

Anti-GAD first detection timing ranged from 1 to 3 years post SPKT (median 1 year). Most anti-GAD+ $(\mathrm{n}=47$, $52 \%$ ) were detectable in the first year of FU screening. Anti-GAD antibodies were detected post-transplant in 90 $(65 \%)$ patients. There were no significant differences between the 2 groups concerning donor/receptor age, IDDM, length of stay, presence of de novo DSA, number of antiHLA, and type/number of mismatches. A trend toward a lower body mass index $(P=.046)$ in anti-GAD + patients was also noticeable. Comparison of annualized mean HbA1c and C-peptide levels according to anti-GAD 
Table 1. Baseline Characteristics According to Recipient, Transplant, and Donor

\begin{tabular}{|c|c|c|c|c|}
\hline & Total $(\mathrm{N}=139)^{*}$ & Anti-GAD+ $(n=49)$ & Anti-GAD-(n= 90) & $P$ Value \\
\hline \multicolumn{5}{|l|}{ Baseline } \\
\hline \multicolumn{5}{|l|}{ Recipient } \\
\hline Age, y, median (IQR) & $35(31-39)$ & $35(31-41)$ & $35(31-39)$ & .465 \\
\hline Female sex, n (\%) & $67(48)$ & $24(49)$ & $43(48)$ & .892 \\
\hline Years of IDDM, median (IQR) & $24(20-28)$ & $24(20-30)$ & $23(20-27)$ & .199 \\
\hline Days of stay, median (IQR) & $15(12-24)$ & $16(12-22)$ & $15(12-24)$ & .730 \\
\hline BMI, median (IQR) & $21.9(20.5-23.7)$ & $22.6(21.1-24.5)$ & $21.6(20.3-23.1)$ & .046 \\
\hline \multicolumn{5}{|l|}{ Transplant } \\
\hline A HLA mistmatch, mean \pm SD & $1.29 \pm 0.60$ & $1.31 \pm 0.62$ & $1.28 \pm 0.60$ & .765 \\
\hline B HLA mismatch, mean \pm SD & $1.64 \pm 0.51$ & $1.65 \pm 0.52$ & $1.63 \pm 0.51$ & .765 \\
\hline DR HLA mismatch, mean \pm SD & $1.42 \pm 0.61$ & $1.41 \pm 0.61$ & $1.42 \pm 0.62$ & .867 \\
\hline Total HLA mismatch, mean \pm SD & $4.35 \pm 1.04$ & $4.37 \pm 1.11$ & $4.33 \pm 1.01$ & .643 \\
\hline Anti-HLA antibodies, n (\%) & $22(16)$ & $9(18)$ & $13(14)$ & .545 \\
\hline Preformed DSA, n (\%) & $11(8)$ & $6(12)$ & $5(6)$ & .163 \\
\hline Pre-SPKT anti-GAD, n (\%) & & & & .001 \\
\hline Negative & $57(41)$ & $29(59)$ & $28(31)$ & \\
\hline Positive & $27(19)$ & $3(6)$ & $24(27)$ & \\
\hline Unknown & $55(40)$ & $17(35)$ & $38(42)$ & \\
\hline Pre-SPKT anti-ICA, n (\%) & & & & .470 \\
\hline Negative & $70(50)$ & $28(57)$ & $42(47)$ & \\
\hline Positive & $15(11)$ & $4(8)$ & $11(12)$ & \\
\hline Unknown & $54(39)$ & $17(35)$ & $37(41)$ & \\
\hline Pre-SPKT anti-IAA, n (\%) & & & & .945 \\
\hline Negative & $13(9)$ & $5(10)$ & $8(9)$ & \\
\hline Positive & $33(24)$ & $11(22)$ & $22(24)$ & \\
\hline Unknown & $93(67)$ & $33(67)$ & $60(67)$ & \\
\hline \multicolumn{5}{|l|}{ Donor } \\
\hline Age, y, median (IQR) & $28(21-38)$ & $26(21-38)$ & $28(21-38)$ & .798 \\
\hline
\end{tabular}

Abbreviations: Anti-GAD, anti-glutamic acid decarboxylase antibody; anti-IAA, anti-insulin antibody; anti-ICA, anti-islet cell antibody; BMI, body mass index; DSA, donor-specific antibody; HLA, human leukocyte antigen; IDDM, insulin-dependent diabetes mellitus; IQR, interquartile range; SPKT, simultaneous pancreaskidney transplant.

${ }^{\star} \mathrm{N}=139$, after exclusion of patients without anti-GAD evaluation post-SPKT.

antibody status is presented in Table 2. Interestingly, an annualized mean higher HbA1c $(P=.006)$ and lower $\mathrm{C}$ peptide levels $(P=.013)$ was evident in anti-GAD + group.

Regarding pre- vs post-SPKT anti-GAD assessment (available for 84 recipients in whom pre-SPKT anti-GAD status was known), 28 converted from negative to positive status, 27 persisted as positive, and 59 were negative, while 3 became negative post-SPKT $(P=.001)$.

Clinical outcomes after transplantation are detailed in Table 3. Alloimmune (ALI) events (pancreas rejection and/ or de novo DSA) occurred in $14(10 \%)$ patients with no differences between anti-GAD+ and anti-GAD- groups $(P=.223)$. Still regarding ALI events, no significance difference was observed in anti-GAD+ and anti-GAD- patients $(P=.419)$ concerning death-censored PG survival.
Patient death occurred in $5(6 \%)$ anti-GAD+ and $2(4 \%)$ anti-GAD - recipients $(P>.999)$.

\section{Auto-immunity: Clinical Associations and Survival}

Forty-two patients showed no ALI events nor anti-GAD antibody positivity, 14 presented with ALI events, and 83 patients reveled AI events (positive for anti-GAD and no ALI events). At 15-year FU, pancreas survival was better in patients with no immune events $(96 \%)$ followed by ALI $(69 \%)$ and AI events $(63 \%)(P=.002$ for ALI and $P=.040$ for AI events in comparison with no immune events) (Fig 1 ). No survival difference was found between patients with AI and ALI events $(P=.192)$.

For the analysis of the impact of pre- vs post-SPKT anti-GAD status on PG survival, we analyzed 74 recipients

Table 2. Annualized Mean of HbA1C and C-peptide in Anti-GAD Positive and Negative Groups

\begin{tabular}{lccccc}
\hline & $\begin{array}{c}\text { Annualized Mean of } \\
\text { HbA1c }(95 \% \mathrm{Cl})\end{array}$ & $\begin{array}{c}\text { Difference } \\
(95 \% \mathrm{Cl})\end{array}$ & $P$ Value & $\begin{array}{c}\text { Annualized Mean of } \\
\text { C-peptide }(95 \% \mathrm{Cl})\end{array}$ & $\begin{array}{c}\text { Difference } \\
(95 \% \mathrm{Cl})\end{array}$ \\
\hline $\begin{array}{l}\text { Anti-GAD } \\
\text { Negative }\end{array}$ & $5.31(5.18-5.44)$ & & .006 & & \\
Positive & $5.53(5.44-5.62)$ & $0.22(0.06-0.38)$ & & $2.91(2.72-3.11)$ & Ref. \\
\hline
\end{tabular}

Abbreviations: Anti-GAD, anti-glutamic acid decarboxylase antibody; $\mathrm{Cl}$, confidence interval; HbA1c, glycated hemoglobin. 
Table 3. Clinical Outcomes After Simultaneous Pancreas-Kidney Transplant

\begin{tabular}{ccccc}
\hline & $\begin{array}{c}\text { Total } \\
(\mathrm{N}=139)\end{array}$ & $\begin{array}{c}\text { Anti-GAD+ } \\
(\mathrm{n}=49)\end{array}$ & $\begin{array}{c}\text { Anti-GAD- } \\
(\mathrm{n}=90)\end{array}$ & $\begin{array}{c}P \\
\text { Value }\end{array}$ \\
\hline $\begin{array}{c}\text { Patient and graft outcomes } \\
\text { Alloimmune events, } \mathrm{n}(\%)\end{array}$ & $14(10)$ & $7(14)$ & $7(8)$ & .223 \\
$\begin{array}{c}\text { Death-censored pancreas } \\
\text { graft failure, } \mathrm{n}(\%)\end{array}$ & $16(12)$ & $4(8)$ & $12(13)$ & .419 \\
\begin{tabular}{c} 
Patient deaths, $\mathrm{n}(\%)$ \\
\hline
\end{tabular} & $7(5)$ & $2(4)$ & $5(6)$ & $>.999$ \\
\hline
\end{tabular}

Abbreviations: Anti-GAD, anti-glutamic acid decarboxylase antibody.

after exclusion of 55 with no pre-SPKT anti-GAD evaluation, 14 with $\mathrm{AI}$ events and a small group of 3 patients with conversion from positive to negative anti-GAD status. Our final sample was then classified into 3 different groups according to pre/post SPKT anti-GAD status (negative/negative, $\mathrm{n}=27$; positive/positive, $\mathrm{n}=22$; negative/positive, $\mathrm{n}=25$ ). Figure 2 shows PG survival by anti-GAD status pre-SPKT and post-SPKT. At 10-year FU (Fig 2), persistent negative, persistent positive, and converted (from negative to positive) recipients showed respectively $100 \%, 88 \%$, and $63 \%$ PG survival $(P=.044)$. In pairwise comparisons, only the difference in survival between persistent negative and converted recipients was significant $(P=.038)$.

\section{DISCUSSION}

Our study about post-SPKT PAs and their relationship with pancreatic graft outcome has a considerable FU time (median of 6 to 7 years) similar to other published cohorts $[13,14]$.

Many SPKT patients have undetectable titers of PAs; others switch from positive to negative titers of antibodies, others maintain stable levels; while others convert from negative to positive. Again, like other studies $[5,9,10]$, we report persistence, disappearance, and reappearance of PAs. In our study, no significant association was observed between post-SPKT ICA and IAA positivity and PG survival. Importantly, the same was not the case for anti-GAD detection post-SPKT, which correlated with decreased PG survival, particularly if the patient had a conversion from negative to positive after transplant.

A recent study found that approximately $7 \%$ to $8 \%$ of all recipients will develop IDDM recurrence, particularly those carrying HLA DR3/DR4 genotype and sharing HLA-DR alleles with the donor $[13,15]$, but other studies did not find this association significant [14]. Similar to this last report, we were not able to discern risk factors for anti-GAD positivity such as HLA mismatch.

Our study observed lower C-peptide levels and higher mean of $\mathrm{HbA} 1 \mathrm{C} \%$ in the positive anti-GAD group. This is consistent with a previous study in which a less favorable glycemic control with a leaning to $\mathrm{HbA} 1 \mathrm{C} \%$ values higher than 5,3\% was observed in patients who developed autoantibody positivity [14]. The association between pancreas autoimmunity and $\mathrm{HbA} 1 \mathrm{C} \%$ and $\mathrm{C}$-peptide directs our attention to the possible role of PAs in monitoring pancreas AI activity. Thus, this suggests that AI should be included in the diagnostic workup of graft failure and ideally should be routinely assessed pretransplant and on FU.

Long-term graft loss may occur because of many factors, including ALI responses (rejection) and recurrence of autoimmunity. In our study, ALI events were present in 14 patients, and AI events (anti-GAD+ but no ALI events) were found in more than $50 \%$ of our cohort $(n=83$ patients). Although our study showed worse PG survival for

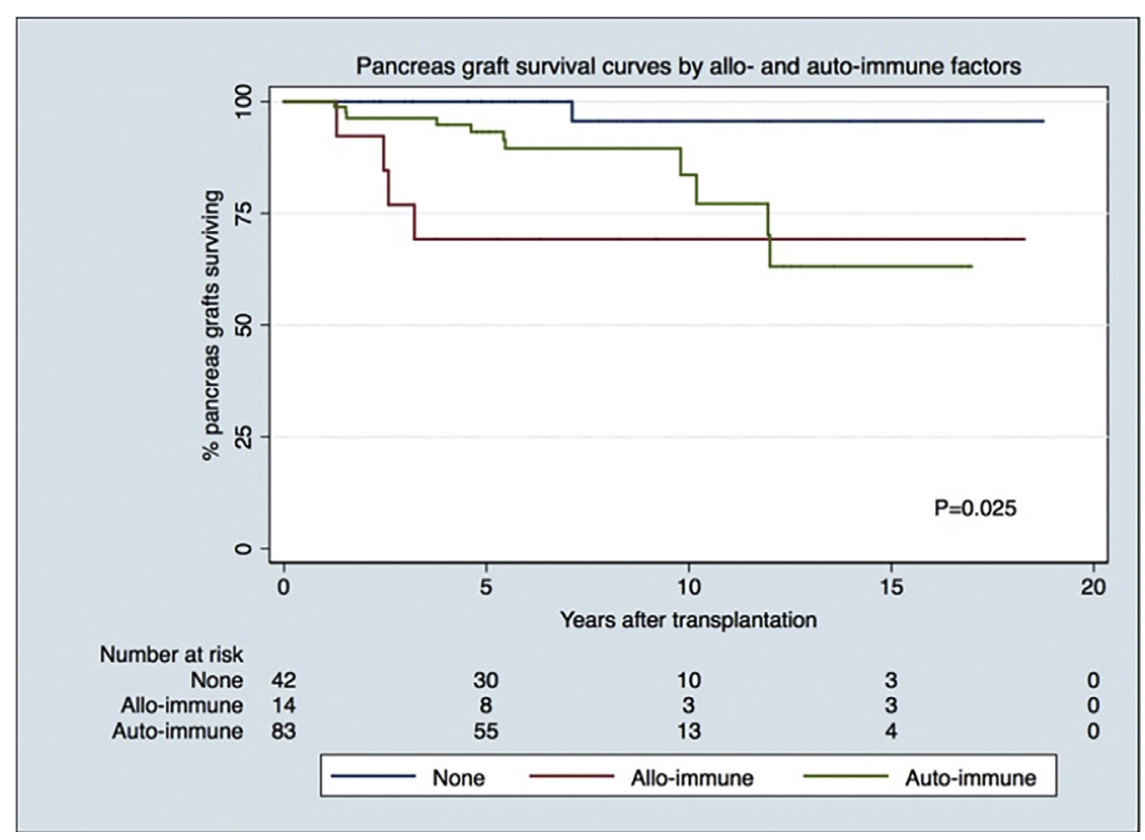

Fig 1. Pancreas graft survival by alloimmune and autoimmune factors. 
Fig 2. Pancreas graft survival by anti-glutamic acid decarboxylase status pre- and post-simultaneous pancreas-kidney transplantation.

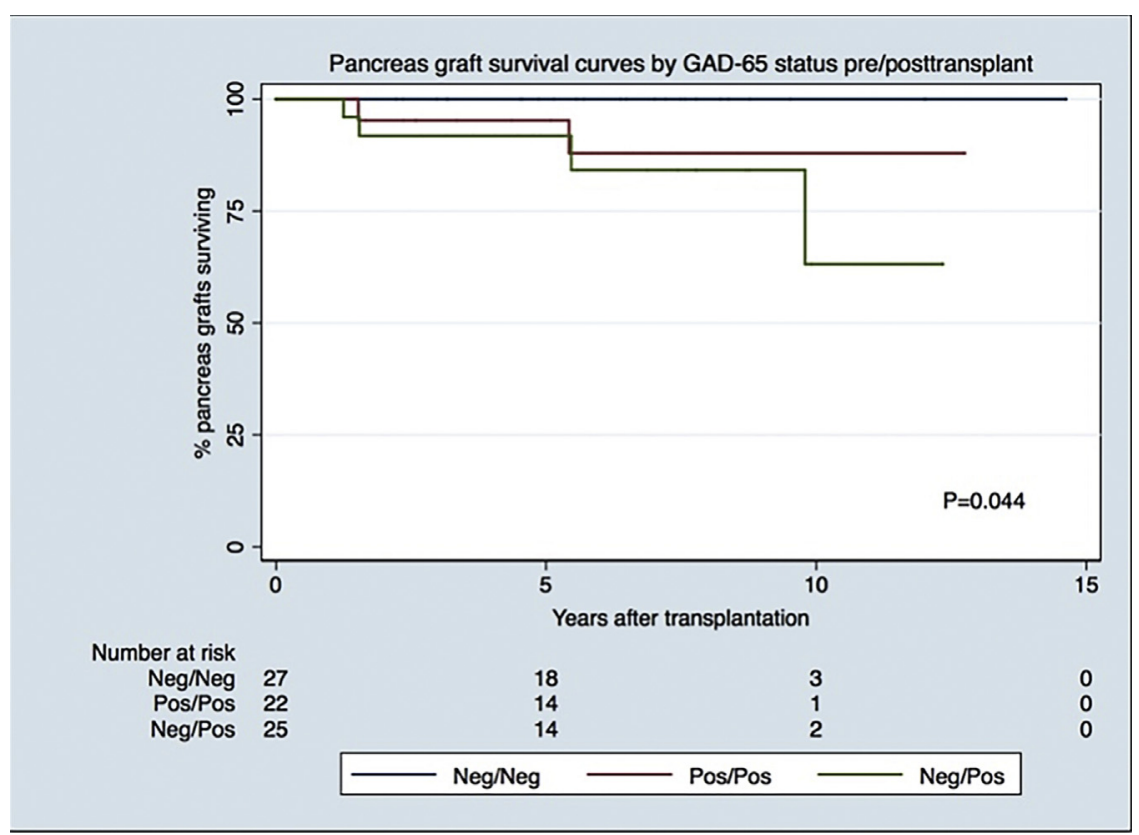

those patients who developed AI or ALI events, no significant differences between them was found. It seems that, despite advances in immunosuppression that prevent rejection, AI and ALI events continue to have a negative impact on PG survival on the long-term.

A key finding, which is confirmed in our study and that extends earlier studies is that autoantibody conversion, as opposed to persistence or negativity, is associated with a high risk of developing IDDM [5,10,15]. Although association between PAs and beta cell destruction seems to be more consistent with anti-GAD antibody in the literature [7], this topic continues to be controversial. In fact, Assalino et al [16] reported a case of confirmed autoimmunity recurrence in which a patient's PAs (GAD-65, IA-2) never converted to positive levels. The real significance and impact of the antibodies on long-term PG survival are still unclear. Our study appears to support findings of other previous published works $[7,10,17]$, in which an association occurs between the recurrence of PAs and poor pancreas survival. At 10-year FU, converted (from negative to positive) anti-GAD recipients showed a $63 \%$ survival compared with those with persistent negative anti-GAD, who revealed a $100 \%$ survival rate. Persistence of anti-GAD positivity did not confer poorer graft survival when comparing with no anti-GAD status pre- and post-transplant.

We recognize that this study has some limitations. First, the high number of missing values of pancreatic antibodies before SPKT hampers a stronger observation of the effect on PG survival of persistence vs conversion antiGAD status. Second, no information about compliance with immunosuppression was available. Last, the absence of histologic confirmation of AI-mediated PG injury phenotype in association with autoantibodies (re-)emergence prevents us from making any causation assumption.

It is believed that pancreatic lesion due to the AI process may be the consequence of the action of complementactivating antibodies targeted at the epitopes of the beta cells or also the result of the lytic action of cytotoxic $\mathrm{T}$ lymphocytes or even the sum of the immunologic response components [18]. It is also known that pathogenic auto reactive CD4 T-cells are associated to B-cell destruction and IDDM recurrence [19]. Further studies will help determine whether assessment of auto-reactive T-cells improves autoantibody prediction. Unfortunately, so far, no current therapeutic regimen has controlled the progression of islet autoimmunity, even when additional immunosuppression was added to the ongoing chronic regimens [15]. The development of new treatment regimens aiming to control autoimmunity is needed, as this may not be effectively suppressed by conventional immunosuppression. Nevertheless, only new and efficacious therapeutic strategies would clearly change the adverse prognosis associated with autoimmunity recurrence.

\section{CONCLUSION}

In conclusion, we consider that our results demonstrate a strong association between post-SPKT anti-GAD antibody detection, PG worse function, and lower survival in SPKT, with this latter effect being particularly noticeable in cases of de novo emergence of this autoantibody after SPKT. 
Hence, longitudinal anti-GAD antibody pattern may improve the clinical management of SPKT recipients, signaling those cases with a higher risk of AI-driven PG lost.

\section{REFERENCES}

[1] Sutherland DE, Sibley R, Xu XZ, Michael A, Srikanta AM, Taub F, et al. Twin-to-twin pancreas transplantation: reversal and reenactment of the pathogenesis of type I diabetes. Trans Assoc Am Physicians 1984;97:80-7.

[2] Sutherland DE, Goetz FC, Sibley RK. Recurrence of disease in pancreas transplants. Diabetes 1989;38(Suppl. 1):85-7.

[3] Bosi E, Botazzo GF, Secchi A, Pozza G, Shattock M, Saunders A, et al. Islet cell autoimmunity in type I diabetes patients after HLA-mismatched pancreas transplantation. Diabetes 1989;38(Suppl. 1):82-4.

[4] Martins L, Malheiro J, Henriques AC, Dias L, Dores J, Oliveira F, et al. Pancreas-kidney transplantation and the evolution of pancreatic autoantibodies. Transplant Proc 2009;41:913-5.

[5] Puigliese A, Reijonen HK, Neopm J, Burke GW 3rd. Recurrence of autoimmunity in pancreas transplant patients: research update. Diabetes Manag (Lond) 2011;1:229-38.

[6] Martins LS. Recidiva da diabetes mellitus pos-transplante de pâncreas. In: Noronha I, Gonzalez A, Meireles Jr R, editors. Manual de Transplante de Pâncreas. São Paulo, Brazil: Segmento Farma; 2011. p. 269-75.

[7] Thivolet C, Abou-Amara S, Martin X, Lefrancois N, Petruzzo P, McGregor B, et al. Serological markers of recurrent beta cell destruction in diabetes patients undergoing pancreas transplantation. Transplantation 2000;69:99-103.

[8] Piemonti L, Everly MJ, Maffi P, Scavini M, Poli F, Nano R, et al. Alloantibody and autoantibody monitoring predicts islet transplantation outcome in human type 1 diabetes. Diabetes 2013;62:1656-64.

[9] Esmatjes E, Rodríguez-Villar C, Ricart MJ, Casamitiana R, Martorell J, Sabater L. Recurrence of immunological markers for type 1 (insulin-dependent) diabetes mellitus in immunosuppressed patients after pancreas transplantation. Transplantation 1998;66:128-31.
[10] Braghi S, Bonifacio E, Secchi A, Di Carlo V, Pozza G, Bosi E. Modulation of humoral islet autoimmunity by pancreas alo-transplant influences allograft outcome in patients with type I diabetes. Diabetes 2000;49:218-24.

[11] Dieterle CD, Hierl FX, Gutt B, Arbogast H, Meier GR, Veitenhansl M, et al. Insulin and islet autoantibodies after pancreas transplantation. Transplant Int 2005;18:1361-5.

[12] Repka J, Nicoluzzi J, Belila R, Antunes M, Caron P. Pancreatic islet cells antibodies in diabetic patients submitted to pancreas transplantation. Transplant Proc 2006;38:3031-2.

[13] Vendrame F, Hopfner YY, Diamantopoulos S, Virdi SK, Allende G, Snowhite IV, et al. Risk factors for type 1 diabetes recurrence in immunosuppressed recipients of simultaneous pancreas-kidney transplants. Am J Transplant 2016;16:235-45.

[14] Martins LS, Henriques AC, Fonseca IM, Rodrigues AS, Oliverira JC, Dores JM, et al. Pancreatic autoantibodies after pancreas-kidney transplantation-Do they matter? Clin Transplant 2014:28:462-9.

[15] Burke GW 3rd, Vendrame F, Virdi SK, Ciancio G, Chen L, Ruiz P, et al. Lessons from pancreas transplantation in type 1 diabetes: recurrence of islet autoimmunity. Curr Diab Rep 2015;15: 121.

[16] Assalinoa M, Genevayb M, Morela P, DemuylderMischlera S, Tosoa C, Berney T. Recurrence of type 1 diabetes after simultaneous pancreas-kidney transplantation in the absence of GAD and IA-2 autoantibodies. Am J Transplant 2012;12: 492-5.

[17] Tydén G, Reinholt FP, Sundkivist G, Bolinder J. Recurrence of autoimmune diabetes mellitus in recipients of cadaveric pancreatic graft. N Engl J Med 1996;335:860-3.

[18] Sutherland DE, Gruessner RW, Dunn DL, Matas AJ, Humar A, Kandaswamy R, et al. Lessons learned from more than 1,000 pancreas transplants at a single institution. Ann Surg 2001;233:463-501.

[19] Vendrame F, Pileggi A, Laughlin E, Allende G, MartinPagola A, Damaris Molano R. Recurrence of type 1 diabetes after simultaneous pancreas-kidney transplantation, despite immunosuppression, is associated with autoantibodies and pathogenic autoreactive CD4 T-cells. Diabetes 2010;59:947-57. 